International Journal of Next-Generation Networks (IJNGN) Vol.2, No.4, December 2010

\title{
AN EFFICIENT VEHICULAR COMMUNICATION OUTSIDE THE CITY ENVIRONMENTS
}

\author{
B. Ramakrishnan ${ }^{1}$, Dr. R. S. Rajesh ${ }^{2}$ and R. S. Shaji ${ }^{3}$ \\ ${ }^{1}$ Associate Professor,Department of Computer Science, S.T Hindu College, Nagercoil-02. \\ Email: ramsthc@gmail.com \\ ${ }^{2}$ Associate Professor,Department of Computer Science and Engineering, Manonmaniam \\ Sundaranar University, Tirunelveli-12. \\ Email: rs_rajesh1@yahoo.co.in \\ ${ }^{3}$ Assistant Professor, Department of Computer Applications, St.Xaviers catholic College of \\ Engineering, Nagercoil. \\ Email: shajiswaram@yahoo.com
}

\begin{abstract}
Communication network plays an important role in providing safe vehicular movement. The main achievement of VANET is providing comfort and safety for passengers. In addition to that, various transactions like information on accident, road condition, petrol bank details, menu in the restaurant, and discount sales can be provided to the drivers and passengers. The speed and time in which the message is sent and received plays an essential part in the Intelligent Transport System (ITS). For this the VANET requires efficient and reliable methods for data communication. Most of the earlier research works deal with the transaction based services provided by road side unit. Moreover the earlier works concentrate on the Intelligent Transport System limited to the city areas where road side units are available in plenty. But this paper discusses the usefulness of VANET outside the city limit. Usually vehicles which move outside the city area do not get the response from the Road Side Unit (RSU) as its availability is limited. For efficient data communication, an attempt has been made to create a new clustering concept among the VANET nodes. The approach of the present paper considers the Simple Highway Vehicular model (SHWM), in which a new clustering model has been introduced. This paper also focuses the service discovery through VANET in the absence of road side units when the vehicles are moving inside the city or outside the city.
\end{abstract}

\section{KEYWORDS}

RSU, OBU, SHWM, DSDV, AODV, DSR AND 802.11P

\section{INTRODUCTION}

A vehicular network is a kind of mobile ad-hoc network. Vehicular ad-hoc network (VANET) enables communication between moving vehicles and the road side units (RSU's) [1]. The use of Wi-Fi, Bluetooth and other mobile connectivity protocols enable data transfer between cars and road side units. The vehicles equipped with an On Board Unit (OBU) will be able to receive and transmit messages [2]. These vehicles with the communicating devices offer a number of benefits and services to the drivers and passengers. For this purpose an Intelligent Transport System (ITS) has been developed that improves the safe travelling with essential alerts and information regarding

DOI : 10.5121/ijngn.2010.2404 
International Journal of Next-Generation Networks (IJNGN) Vol.2, No.4, December 2010

entertainments [3]. VANET is mainly used to provide a communication model in a vehicular environment where the vehicles are considered as VANET nodes with wireless links. Vehicles move in high speed or low speed in the highways. The movement of the vehicle is dependent on the road condition such as narrow, curved and traffic congestion. The high speed vehicles form a quick dynamic network topology in which a real time data transmission takes place. These characteristics of vehicular network necessitate creation of a new vehicular architecture, data communication protocols and standards. As on date only a few VANET surveys are made and these are mostly dealt with the vehicular communication inside the city where enough road side units are present [4]. No major study has been made on the highway vehicular movement without RSUs. For this reason this paper focuses a new vehicular model for data communication outside the city [5][6].

The second chapter describes the previous works related to Mobile Ad-hoc network communication. The third chapter discusses the limitation of the existing model and how to overcome the limitations. The next two chapters namely 4 and 5 deal with the development of a new cluster based model and how the new model differs from the pervious one. The chapter 6 describes a suitable simulator, software and platform for carrying out this analysis. The chapter 7 defines some parameters to measure the efficiency of the data communication in the proposed model and presents a brief summary of the experimental results. The last chapter gives the conclusion.

\section{RELATED WORK}

VANET is an advanced version of Mobile ad-hoc network (MANET). Most of the MANET features can be applied in the VANET environment also [7]. In VANET the vehicles move in an organized and predefined road. In MANET the movement of the mobile nodes is at random. Unlike mobile ad-hoc network, there is no power constraint for vehicles in a vehicular network. VANET is the special type of MANET, so the routing Protocols and IEEE standards used in MANET are also applied in VANET Environment [8][9]. In MANET, the nodes are moving at random and their speed is normal. For the data communication in mobile ad-hoc network, the technology IEEE 802.11 is used [10]. Most of the existing research works have been done on MANET. However these works cannot be directly applied to VANET due to the fundamental difference between VANET and MANET. The literature survey available on vehicular communication is very limited and most of them are the extension of the mobile ad-hoc networks. In vehicular network, the mobility nodes are vehicles which are moving in a high speed of nearly $200 \mathrm{~km} / \mathrm{hr}$ on a predefined road. The movement of nodes is dependent on the road structure, traffic and traffic regulation. Due to this high speed of the vehicles the usual mobile ad-hoc technology IEEE802.11 is not well suited for VANET. For this reason a suitable amendment is made on the existing standard 802.11 that becomes a new vehicular technology 802.11p. Another big challenge is creating the vehicular network outside the city area. Most of the existing research works are on the vehicular motion within the urban area. But the real issue is to develop a model for the highway mobility outside the city. Therefore a new highway mobility model is developed with a new cluster concept that increases the efficiency of the data communication.

\section{Limitation Of The Existing Model}

\subsection{Using the IEEE standard 802.11.}

In the MANET, the nodes are moving at random and their speed is normal but in the VANET, nodes are high speed moving vehicles. Due to the high speed of the vehicle nodes, their network 
International Journal of Next-Generation Networks (IJNGN) Vol.2, No.4, December 2010

topology changes very quickly. Hence the technology 802.11 used in MANET yields less performance in VANET. So the IEEE working group has developed a new PHY/MAC amendment of the 802.11 standard which is specially designed for vehicular communication. This wireless access in vehicular environment (WAVE) is known as IEEE 802.11p which is more suitable for high speed vehicle data communication [11]. This amendment is based on the study of the vehicular safety concepts, communication between vehicle and vehicle, and vehicle to road side unit. The most important parameters for a VANET MAC protocol are low latency and high reliability. The performance of the 802.11 over the $802.11 \mathrm{p}$ had already been discussed by the author [12]. Most of the earlier vehicular research works have been done on the standard 802.11 only but in this paper an attempt has been made to introduce a new VANET based technology with a new highway model. This vehicular model with $802.11 \mathrm{p}$ standard is simulated using the NS2.34 version simulator. The IEEE $802.11 \mathrm{p}$ technology can be used in NS2.33 and above versions only.

\subsection{Using road side units}

When a survey is made on the subject, it is found that the protocols and mobility models are limited. So various attempt have been made to create a new mobility models and routing protocols for VANET. Another big challenge is creating a suitable vehicle movement model for cities and the surrounding areas. Most of the researchers have dealt with the vehicular motion inside the city limit only [13]. The real thrust is to find the highway mobility model outside the city. Roadside units are more in the city areas whereas they are less in outside city areas. So a different architecture is necessary to solve the problem related to the road side unit. Hence a new cluster based VANET model for highways is created.

\section{Data Communication In Vanet}

A routing protocol plays an essential role in vehicular network data communication. The routing protocols included in NS2.34 version are Destination Sequence Distance Vector (DSDV) protocol, Dynamic Source Routing (DSR) and Ad-hoc On-demand Distance Vector (AODV) routings protocol. This paper analyzes the performance of data communication with DSDV routing protocol [14]. The type of data communication in the vehicular network can be classified into two main categories

i. Broadcast application services.

ii. Transaction based services.

A broadcast service is used to inform the drivers about an emergency situation. The data rate is not an important issue here because each vehicle wants to receive only warning alert messages [15]. The transaction based service is used to provide location based services such as information about petrol station, police station or hospital information etc [16]. In this proposed model three types of data communication in the vehicular network are discussed.

\subsection{Data communication with road side units}

Road side units are more in the city areas whereas they are less in outside the city and less populated areas. When a vehicle enters a city area, it directly sends the request to the road side units and gets the responses easily. The vehicle does not get response from the outside areas because of the limited availability of Road side units. In the earlier vehicular network model all the nearby vehicles are connected to this road side units and all the road side units are in turn connected together to form mobile ad-hoc network which is shown in Figure 1. This scenario is valid only for 
the vehicles moving inside the city where the vehicles move slowly and more number of fixed base stations is available.

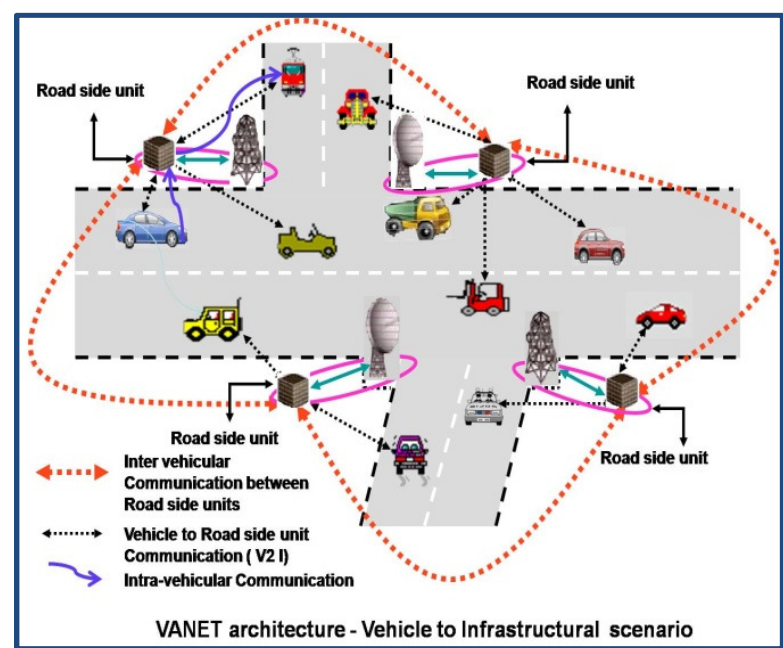

Figure 1: Vehicular architecture with road side units

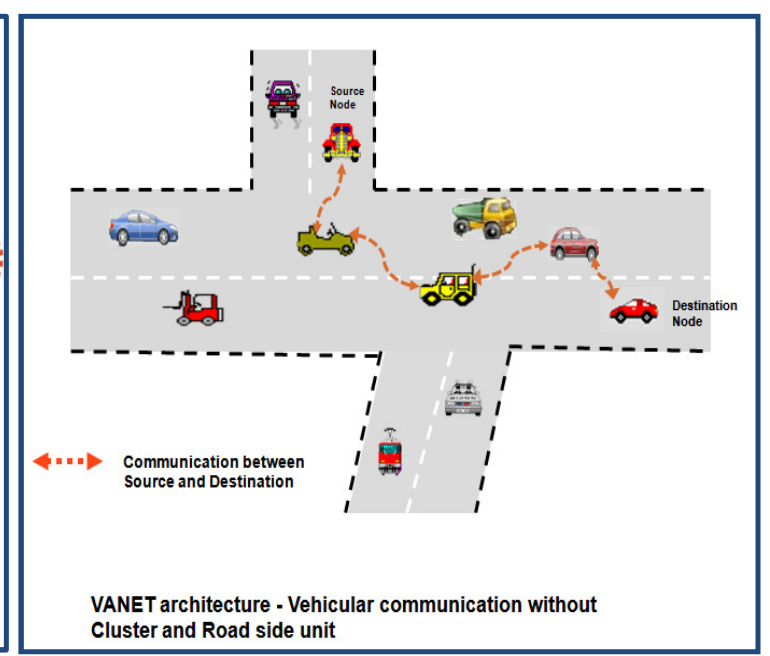

Figure 2: Vehicular architecture without using RSU'S

\subsection{Data communication without roadside units}

In suburban and unpopulated areas, only a few or no roadside units are available for data communication. The vehicles in the outside city area do not get response because of the limited availability of road side units and the high speed of the vehicular movements. So various attempt have been made to create a new mobility model and routing protocols for VANET. This paper presents two approaches for data communication outside the city limit where there are no road side units.

\subsubsection{A simple highway model without using road side units and cluster concepts.}

This paper analyzes the existing model and suggests a new method to solve the problem related to the road side unit. Vehicles moving on either direction in a highway have a limited radio range. The vehicle within a radio coverage range in the new model can communicate directly with other moving vehicles whereas the same type of communication happens through a fixed road side unit in the existing model. In the previous models the roadside units act as a router to communicate the information from source vehicle to destination vehicle. But in this proposed model the data packets can be delivered from source vehicle to destination vehicle without using RSUs. Here each vehicle acts as a router. The node communicates to the destination using multi-hop communication. The data communication architecture without using cluster concepts and road side units is shown in Figure 2.

\subsubsection{A simple highway model using cluster concepts without road side units}

A new cluster based highway architecture is presented here that increases the efficiency of the vehicular communication such as data transfer from source to destination, service request from any node in the network and broadcasting warning messages to all VANET nodes. 
International Journal of Next-Generation Networks (IJNGN) Vol.2, No.4, December 2010

\section{Simple Highway Mobility Model (ShWM)}

In the new concept a simple highway system is taken for characterizing the VANET. On highways, vehicles can usually move freely on either direction. Each vehicle can have a limited radio range. A Vehicle within a radio coverage range can communicate directly whereas it takes place through a fixed roadside unit in the existing model. The newly proposed system takes into consideration the two scenarios:

1: Service Discovery when a vehicle moves inside the city.

2: Service Discovery when a vehicle moves outside the city.

In the earlier model, a road side unit is used as a router to communicate the messages. Here all the nearby vehicles are connected to the road side units to form a mobile ad-hoc network. The intravehicular communication from vehicle to vehicle through the RSU's and inters-vehicular communication through the RSUs are shown in Figure 1. In the non-urban area, limited road side units are available for data communication. For effective vehicular communication each vehicle acts as a router to exchange information. Each vehicle is equipped with a global positioning system (GPS) [17]. Broadcasting and routing algorithms use the information provided by the GPS and make effective communication. For the road safety, new applications are proposed for vehicular networks, i.e. car to car communication, travel and tourism information distribution and game applications. These applications need reliable communication equipment with high data rates and also a stable connectivity between the transmitter and the receiver under high reliability condition. The vehicular communication with cluster concepts is shown in the Figure 3.

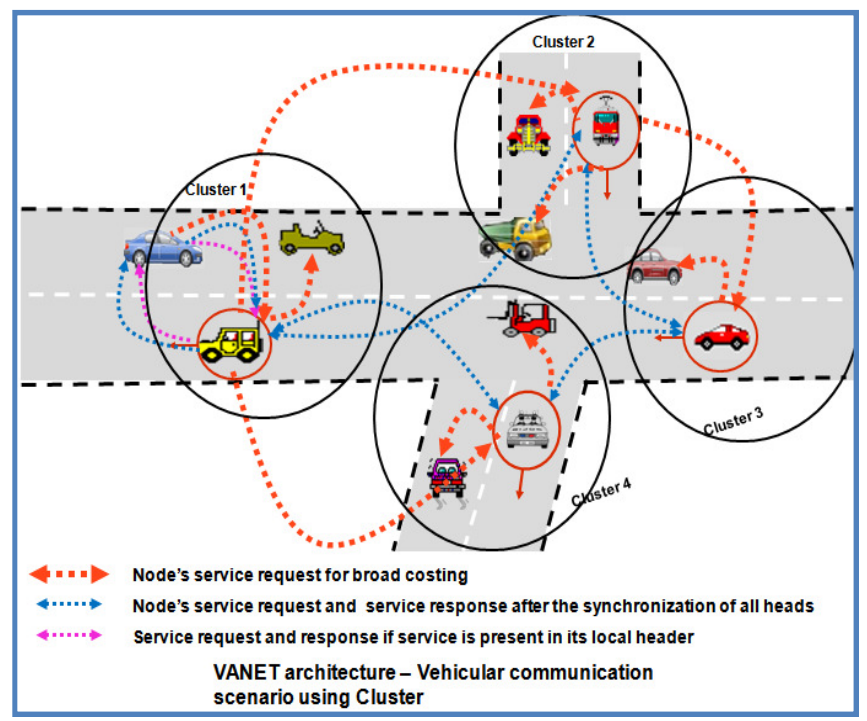

Figure 3: Vehicular architecture with clusters

\subsection{Cluster Based VANET Architecture}

The Cluster creation process in the proposed model is different from the existing model [18]. In MANET, clusters are created dynamically but in the VANET the clusters remain fixed and predefined [19]. The size of the cluster changes only during unavoidable situations like sudden increase in the number of vehicles moving in a particular road due to traffic changes. In the proposed system, the cluster remains in the same frequency. Unlike MANET, the VANET nodes 
International Journal of Next-Generation Networks (IJNGN) Vol.2, No.4, December 2010

move on predefined paths mostly in roads only. So the cluster areas are created as fixed ones. But the cluster creation process involves a series of important steps to ensure that the node in the created cluster is efficient so as to give reliable communication in service discovery [20]. While creating a cluster, it should be ensured that the cluster head does not frequently cross the cluster boundary. If the vehicle moves out frequently, the Cluster head election algorithm often elects a new cluster head. The cluster creation, cluster head election and cluster head switching procedure for highway model are discussed by author [21].

In the present architecture, the VANET area has been split into a number of clusters by using the proposed cluster formation algorithm. Each cluster has a cluster head. The cluster head may be either RSU or any one of the vehicles with good database storage and access capabilities. This cluster head is selected by the new cluster head algorithm proposed in this model. Each cluster head has all the service descriptions that are available in the network. All the cluster heads in the VANET are regularly updated if a new service enters in the network. All the cluster heads are synchronized in a specific time interval. The cluster heads are synchronized to ensure that the cluster heads have same value. Cluster concept has successfully been applied in MANET for a better delivery ratio and to reduce broadcast storms. In a Cluster environment, the Cluster head receives information from any node of that cluster and sends it to another cluster head. Cluster-based solutions provide less propagation delay and high delivery ratio. Clustering can simplify such essential functions as routing, bandwidth allocation, and channel access. Several heuristic clustering techniques have been proposed to choose cluster heads in MANET. These procedures are also applicable to VANET environment.

\subsection{Cluster Creation Process}

If cluster heads frequently change their relative position on highways, the size and stability of clusters change unpredictably. Vehicles on highways usually have the same direction. Therefore, their geographical location and speed information are helpful in the cluster creation process. Better performance can be achieved, if the geographic positions of the network nodes are known. Clusterbased solution is a viable approach in supporting efficient multi-hop message propagation among vehicles. The present algorithm follows two strategies while creating a cluster area. If the nodes are moving slowly and the node density is high, then the algorithm creates a medium size cluster. If the node density is high, traffic is heavy and road condition is poor then the vehicular movement is slow so the cluster area is chosen to be small. If the cluster area is small, there is a possibility for the node to reach its cluster boundary very quickly and the efficiency of the network is low. When the density of the vehicles is less, the speed of the vehicle will be high. In the new system the algorithm creates a large size cluster. When the vehicle movement is slow in the cluster area, then the cluster head switching may not occur frequently. Care should be taken to keep the cluster area as a medium size for efficient management which ensures the cluster head switching may not occur frequently. Previously, the services of VANET were provided by the road side units which acted as a router. In the present system, the VANET is approached in the form of clusters. Each vehicle in the cluster acts as a router. The present paper focuses on Service announcement and Service discovery in VANET.

\subsection{Cluster Creation Algorithm}

In this algorithm, ' $n$ ' is the total number of nodes, ' $\mathrm{i}$ ' is the node that is varying from 0 to $n-1$. If $n$ is equal to 3 , then the values become $\mathrm{x}_{0} \mathrm{y}_{0}, \mathrm{x}_{1} \mathrm{y}_{1}, \mathrm{x}_{2} \mathrm{y}_{2}$. This algorithm tracks the position of the node in 
the cluster areas. $\left[\mathrm{A}_{\mathrm{i}}, \mathrm{B}_{\mathrm{i}}\right]$ is the subset of $\left(\mathrm{X}_{\mathrm{i}}, \mathrm{Y}_{\mathrm{i}}\right)$ that contains the $(\mathrm{X}, \mathrm{Y})$ position of the node of a particular cluster area.

This algorithm finds the speed movement of $\mathrm{N}[\mathrm{k}]$ that belongs to $\left[\mathrm{A}_{\mathrm{i}}, \mathrm{B}_{\mathrm{i}}\right]$. If the average speed of the vehicle is low, then this algorithm takes a small cluster size. Otherwise it takes a large cluster size. The function used in this algorithm returns $(\mathrm{X}, \mathrm{Y})$ position of each individual node.

Algorithm 1: The cluster creation algorithm for the proposed SHWM

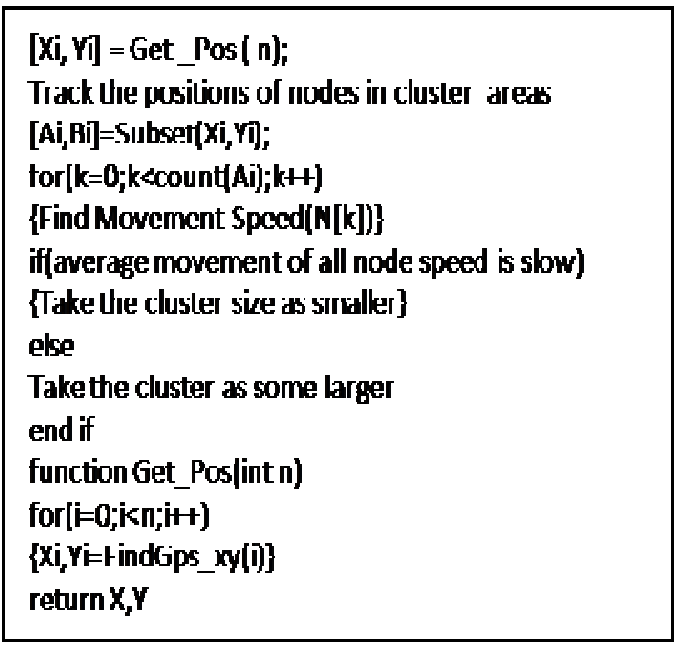

\subsection{Service Announcement}

The exchange of information in the Service Announcement procedure takes place among the cluster heads. Only the cluster heads receive the service information and update their local database. If a new service is introduced, then all the cluster heads are immediately updated with the new information or data. If a node introduces any service, then it informs the local cluster head about it. The local cluster head multicasts the service description to all other cluster heads. The cluster head receives the service description and stores it in the local database.

Algorithm2: Broadcasting new services

While(true)
Begin While
If a node introduce a new service
Begin If
Find all the cluster heads in the network
Multicast the new service information
to all the Cluster heads
End If
End while




\subsection{Service Discovery Approach}

If a node in a cluster wants to search a service, then initially it contacts its local Cluster head. The local cluster head searches in the local database whether the specified service is available or not. If the specified service is available, then it will give the necessary details about the service provider to the needed node to get the service. If the service is not available, then the algorithm synchronizes all cluster heads in the VANET immediately. After synchronizing the procedure, again it searches the cluster head for the availability of the required service. Again if the service is not available, it just informs the needed cluster service node that the service is not available.

Algorithm 3: Data communication algorithm for SHWM

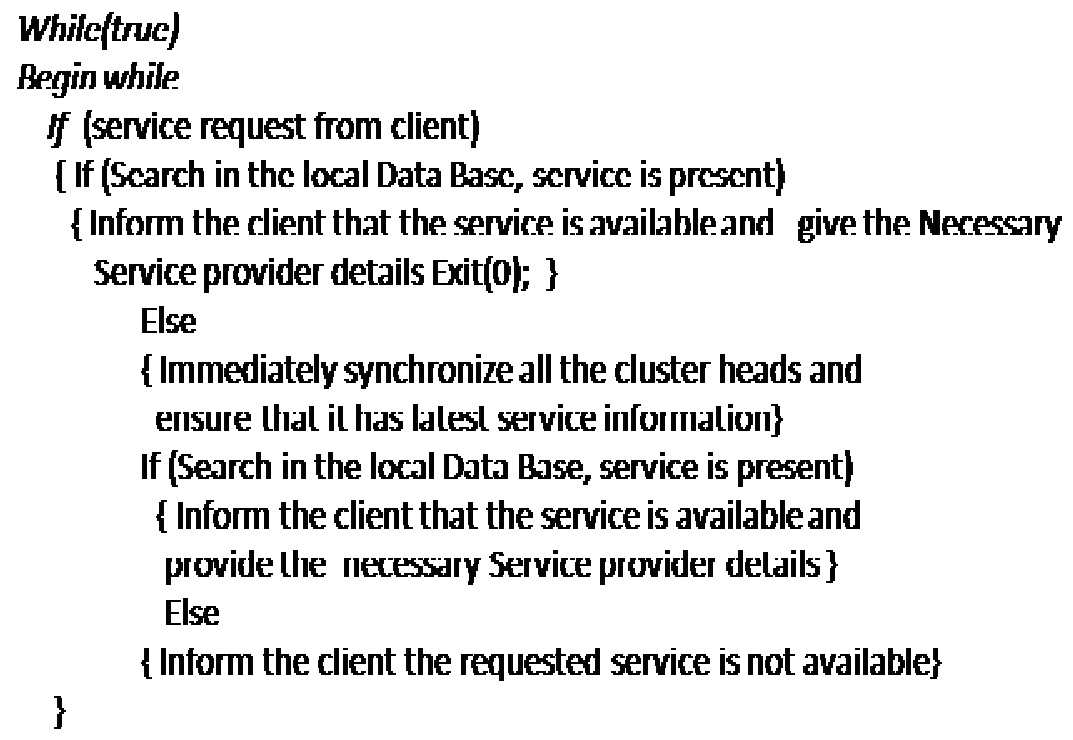

\section{Simulation SetTing}

The Cluster based simple highway mobility model has been thus proposed and simulated by using NS 2.34 version on Linux redhat 9. The following parameters are used in this test bed. VANET area is assumed as $1500 * 1500$ meters of highway with bidirectional movement of Vehicles. Number of Nodes varies between 25 and 150. Number of Clusters varied between 2 and 20 in steps of 2. Speed of the vehicles is assumed to be constant in this paper between $5 \mathrm{~m} / \mathrm{s}$ and $25 \mathrm{~m} / \mathrm{sec}$. The standard $802.11 \mathrm{p}$, which is well suited for VANET communication is adapted in this scenario. This paper analyses the performance of Packet receiving time, Packet delivery ratio, Normalized routing load, Broadcasting time and Delay time for different nodes with various clusters and speeds. The paper also focuses on the performance analysis of standard 802.11p over 802.11. To enable reliability, for each category of result, averagely 50 samples have been taken. 
International Journal of Next-Generation Networks (IJNGN) Vol.2, No.4, December 2010

\section{Simulation Result ANd Analysis}

To increase the efficiency of the highway data communication, new cluster concept and 802.11p technologies are included in this model and their characteristics are given below. The Routing protocols used in this highway model is discussed by the author [9]

\subsection{Packet receiving time of the Cluster based SHWM model with DSDV and 802.11p}

The Figure4 shows the packet receiving time for cluster eight with varying nodes between 25 and 150 and the packet receiving time for without cluster concept for node varying from 25 and 150 with using VANET standard IEEE 802.11p. From the graph, the number of nodes below 100 yields low packet receiving time and almost constant value irrespective of the number of nodes above 100. The packet receiving time for standard $802.11 \mathrm{p}$ with cluster concept is better than without cluster concepts.

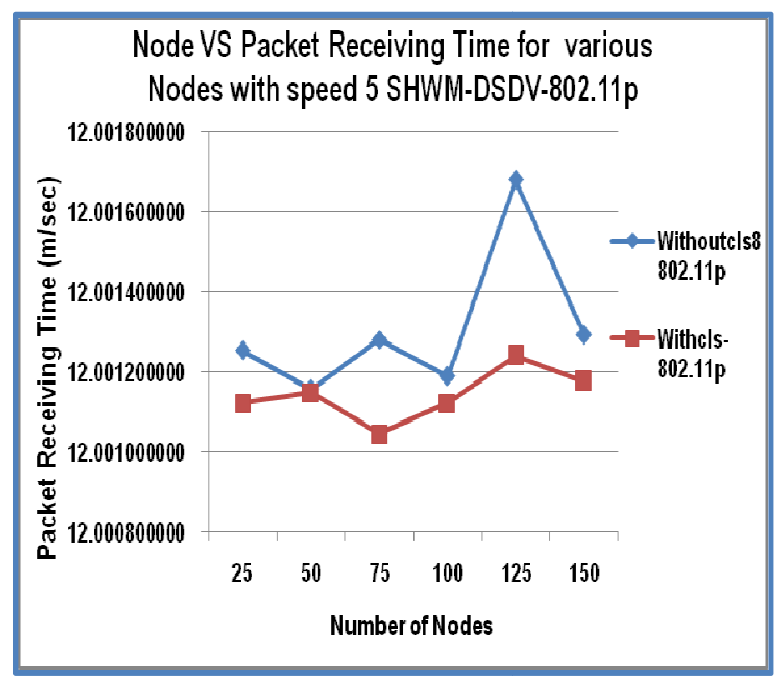

Figure 4: Nodes VS Pkt Receiving Time ( $\mathrm{m} \mathrm{sec}$ ) with \& without cluster concept

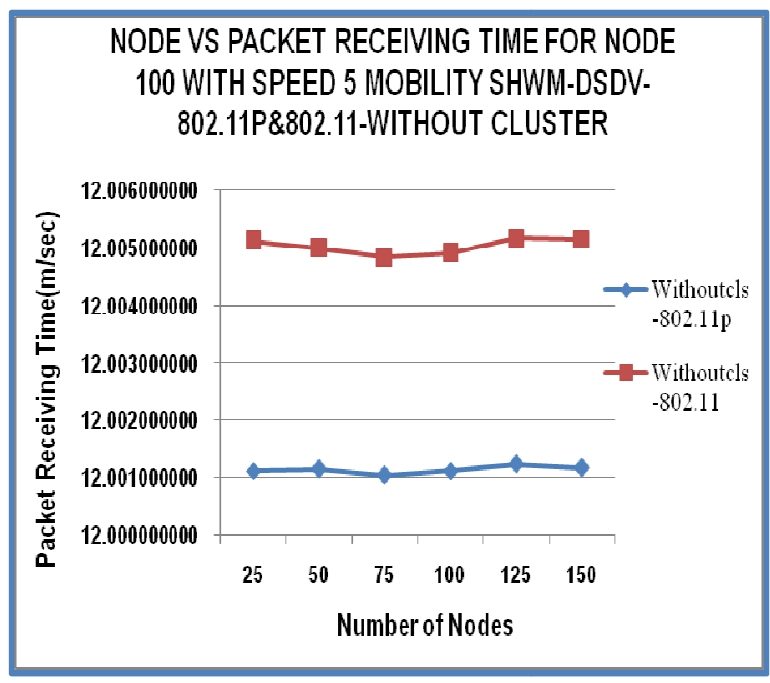

Figure 5: Number of nodes VS Pkt Receiving Time with 802.11 p \& 802.11 without cluster

\subsection{Comparison of Packet receiving time for 802.11p and 802.11}

From Figure 5, the packet receiving time of the destination node is noticed in the highway model in which the number of nodes varying from 25 to 150 with MAC 802.11 and 802.11p. Without using the cluster concepts, it is observed that the packet receiving time is high for 802.11 and it is low for 802.11p. No major change is observed when the number of node is increased.

\subsection{Comparison of Packet receiving time for 802.11p with 802.11 using cluster concepts}

The Packet receiving time for number of clusters with IEEE standard 802.11 and $802.11 \mathrm{p}$ is presented in this Figure 6. It is observed that the performance of the standard 802.11p with DSDV protocol is better than the 802.11 technology. The repeated experiment has proved the above observation. 
International Journal of Next-Generation Networks (IJNGN) Vol.2, No.4, December 2010

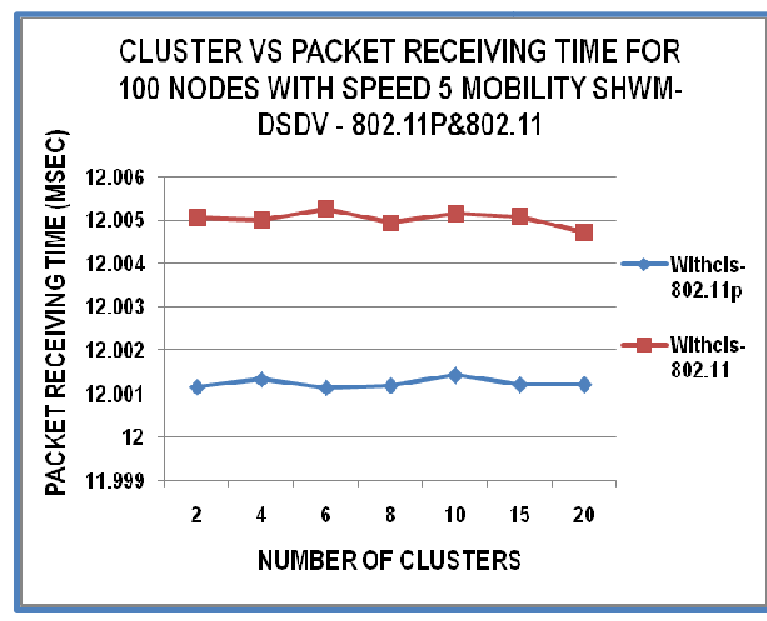

Figure 6: Cluster VS Packet Receiving Time ( $\mathrm{m} \mathrm{sec}$ ) with cluster

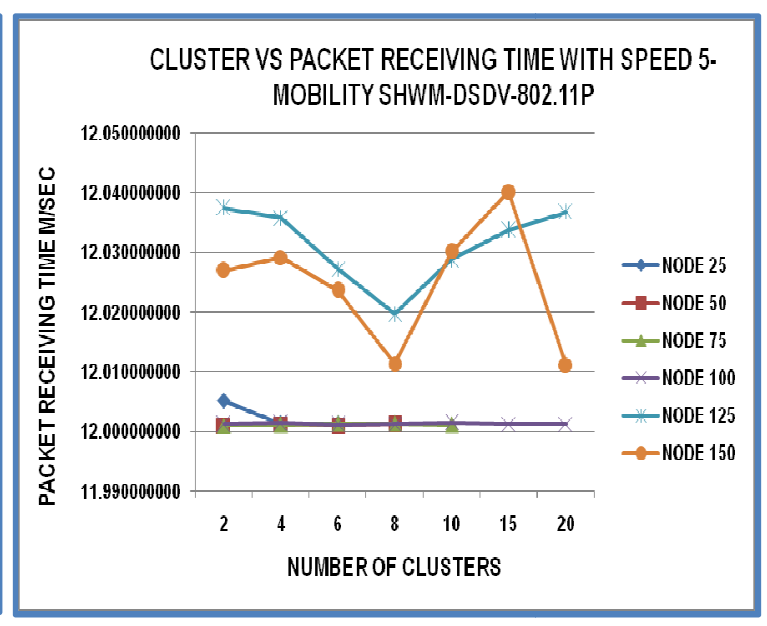

Figure 7: Performance of Pkt Receiving Time for cluster varies between 2 and 20

\subsection{Analysis of Packet receiving time for various clusters using 802.11}

In Figure7, the packet receiving time for various clusters with various numbers of nodes is mentioned. In this process the packet receiving time is quick as the number of nodes are 100 and below 100. The performance would be worse, if the number of nodes increases from 100.

\subsection{Broadcasting time for the Cluster based SHWM model with DSDV and 802.11p\& 802.11}

The performance of the Cluster based SHWM model with $802.11 \mathrm{p}$ is compared with the same Cluster based SHWM model with 802.11 which is shown in figure 8. The Broadcasting time of packets to all cluster head is estimated for various clusters. The proposed cluster based SHWM with 802.11p outperforms the SHWM with 802.11. The Broadcasting time slightly increases in 802.11p when the number of clusters increases. But in 802.11, the change would be drastic. From Figure 9 it is clear that when the number of nodes increases the packet receiving time decreases. It is further noted that better broadcasting time is received when the number of clusters increases.

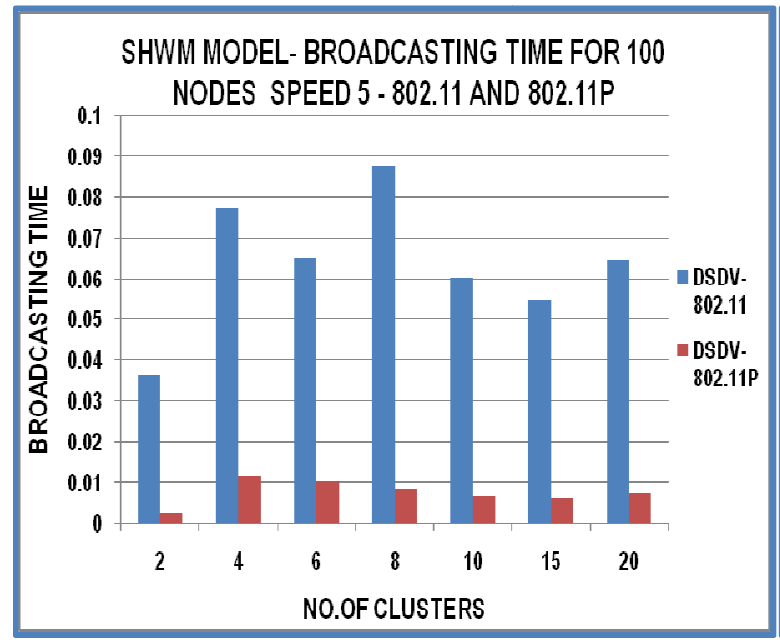

Figure 8: Cluster VS Broadcast Time (msec)

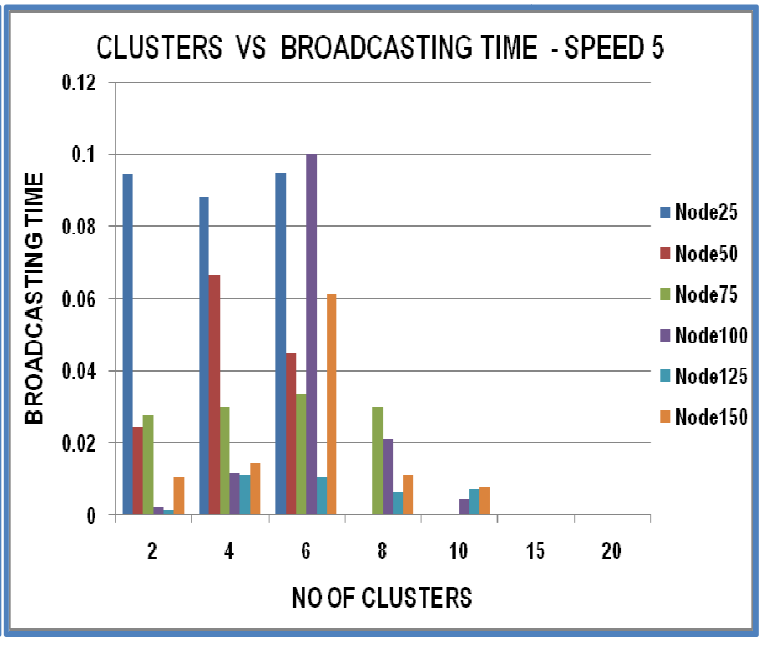

Figure 9: Clusters VS Broadcast Time(msec) 


\subsection{Root request time Vs Number of Clusters}

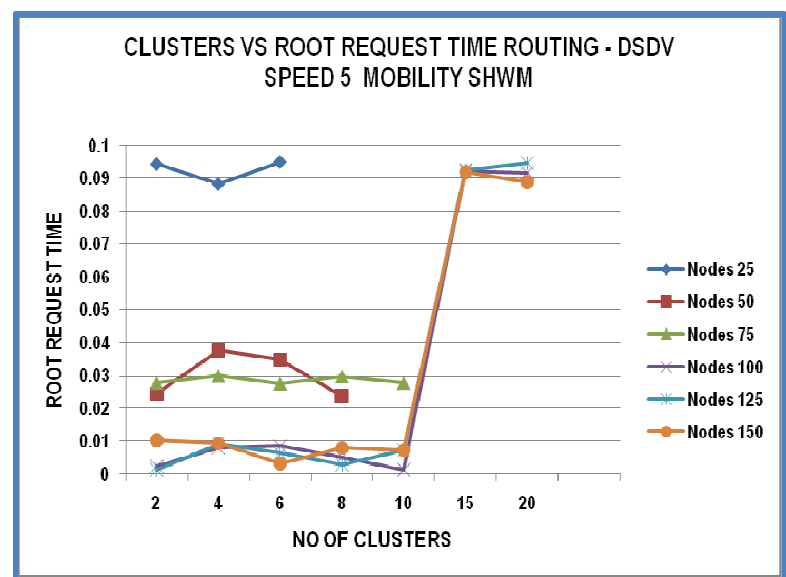

Figure10: Analysis of RRT Vs cluster

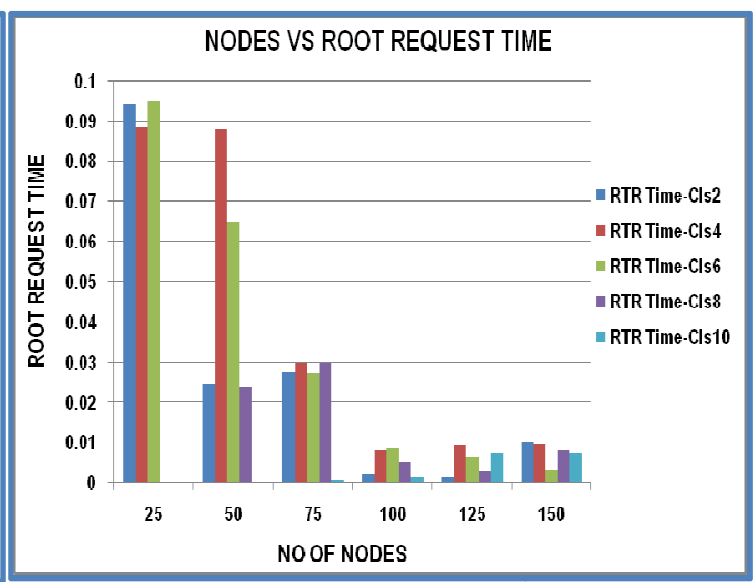

Figure 11: Analysis of RRT Vs Nodes

The efficiency of the Root Request Time for different clusters and various nodes is listed in Figure 10 and 11. The RRT is high when the number of node is 25 ; the RRT is moderate when the number of nodes falls between 50 and 75; whereas the RRT is low, when the number of nodes is between 100 and 150.

\subsection{Throughput of the Cluster based SHWM model with DSDV and 802.11p.}

The performance of SHWM in terms of throughput is shown in Figure 12. The graph clearly describes the cluster Vs throughput for various clusters with nodes between 25 and 150 with speed 5. The protocol and standard used in this simulation are DSDV and $802.11 \mathrm{p}$ respectively. From the test bed result, it is noticed that when the number of nodes increases, the throughput decreases. The 25 nodes have maximum throughput value. It is also observed that when the number of clusters increases from 2 to 20 in multiples of 2 , the throughput value slightly changes and no major change is traced there.

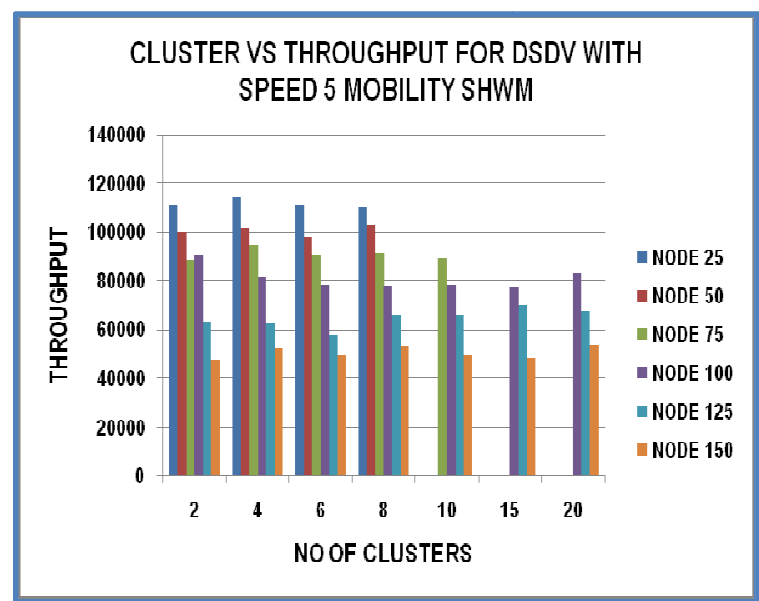

Figure 12: Cluster VS Throughput for Nodes 25 to 150

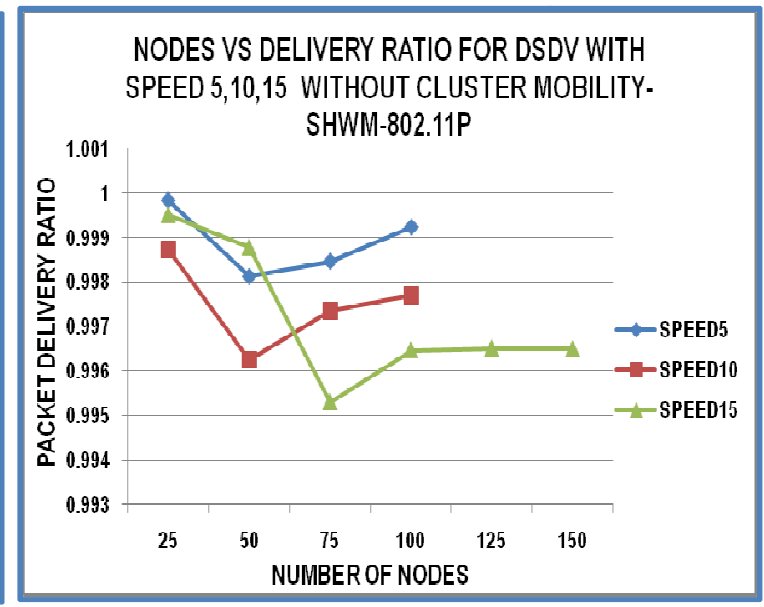

Figure 13: Cluster VS Packet Delivery ratio for speed 5 to $15 \mathrm{~m} / \mathrm{sec}$ 
International Journal of Next-Generation Networks (IJNGN) Vol.2, No.4, December 2010

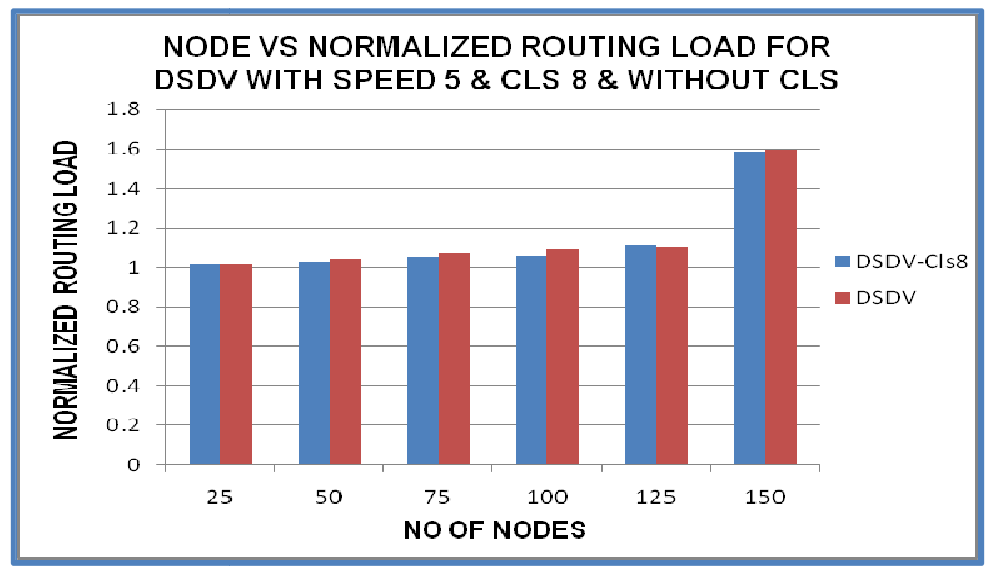

Figure 14: Nodes VS Normalized routing load for speed 5 to 25

\subsection{Packet delivery ratio for the Cluster based SHWM model with DSDV and 802.11p}

Figure 13 presents the packet delivery ratio for various nodes with different node speeds. From this graph, it is observed that the packet delivery ratio obtained for node 25 to 150 with speed 5 yields better performance than speed 10 and speed 15.

\subsection{Normalized routing load for the Cluster based model with DSDV and 802.11p}

The Normalized routing load for various nodes with cluster and without cluster concepts is estimated in Figure 14. Better performance is confirmed when the cluster concept is used. No other significant change is visible when the DSDV protocol is experimented.

\section{Conclusion And Future Direction}

VANET is a component of MANET. Most of the existing research work is done on MANET. As on date the literature available for VANET is very limited, mostly extension of MANET concepts. Most of the researchers use standard 802.11 for VANET model with low movements of mobile nodes within the city area. But this technology is not well suited for the vehicles moving with a very high speed nearly $200 \mathrm{~km} / \mathrm{hr}$. Due to this high speed the network topology changes quickly. So an amendment to 802.11 is made and it is known as $802.11 \mathrm{p}$. This new one is used in the highway model. This new VANET technology is used this scenario to analyze the performance of the data communication with Routing DSDV. Only NS2.33 and above version is used to simulate the IEEE $802.11 \mathrm{p}$ standard. Here the latest version NS2.34 is used to analyze the performance of the data communications. The simulation result gives that the proposed model with $802.11 \mathrm{p}$ has outperformed the result obtained using 802.11. The proposed new SHWM model with Cluster concepts and the standard 802.11p outperforms the existing models that use roadside units.

To increase the efficiency of the data communication in the highway model a cluster concept is introduced in the newly developed simple highway model in which each cluster head acts as a router to communicate with the other vehicles. For normal service discovery methods, (n-1) searches are required to find out the destination vehicle. Even if the node is not available in the VANET, the search may be continued with all possible nodes in the vehicular network. But in our new methods only the head is searched to find out the availability of the destination nodes. It is also noticed that the warning message is initially sent by the node to its local cluster head. The local cluster head then broadcasts this message to all the cluster heads. Then these cluster heads in turn 
International Journal of Next-Generation Networks (IJNGN) Vol.2, No.4, December 2010

communicate this emergency message to all its connected vehicles simultaneously. This model provides an efficient architecture for service request/response and broadcasting the warning messages.

The earlier VANET models discuss only the communication between vehicles through the RSU. But this paper I hope, has clearly discussed the highway model with latest VANET standard 802.11p and new cluster concepts, which will pave the way for developing new Routing Protocols, Mobility Models and IEEE technologies in future.

\section{REFERENCES}

[1] Lin Yang Jingdong Xu Gongyi Wu Jinhua Guo, Nankai Univ Tianjin, China "Road Probing: RSU Assisted Data Collection in Vehicular Networks" Wireless Communications, Networking and Mobile Computing, Beijing, page: 1-4

[2] Wern-Yarng Shieh Wei-Hsun Lee Shen,"Analysis of the Optimum Configuration of Roadside Units and Onboard Units in Dedicated Short-Range Communication Systems", Intelligent Transportation Systems, IEEE Transactions on Issue Date : Dec. 2006 Volume : 7 , Issue:4 On page(s): 565 - 571

[3] Biswas, S. Misić, J. "Proxy signature-based RSU message broadcasting in VANETs", Communications (QBSC), 2010 25th Biennial Symposium on Date: 12-14 May 2010 On page(s): 5 - 9 Location: Kingston

[4] Wenping Chen "VANETs-based real-time traffic data dissemination ", Wireless Communications, Networking and Information Security (WCNIS), 2010 IEEE International Conference on Issue Date : 25-27 June-2010,page(s): 468-472Location: Beijing,China.

[5] Zhang, M. Wolff, R. "Routing protocols for vehicular Ad Hoc networks in rural areas" This paper appears in: Communications Magazine, IEEE - 2008, Volume : 46, Issue:11 On page(s): 126 - 131 -Canada.

[6] Fang, Y.M. "Vehicular Ad Hoc networks: Communications on the road ", wireless communication IEEE Issue, Date: December 2009 Volume: 16, Issue6 On page(s): 2 - 3.

[7] Saxena, N.; Tsudik, G.; Jeong Hyun Yi; Polytech. Univ., Brooklyn"Efficient Node Admission and Certificateless Secure Communication in Short-Lived MANETs",Parallel and Distributed Systems, IEEE Transactions - Feb. 2009, Volume: 20 Issue:2 On page(s): 158 - 170.

[8] Tuteja, Asma Gujral, Rajneesh Thalia, Sunil," Comparative Performance Analysis of DSDV, AODV and DSR Routing Protocols in MANET Using NS2", ACE, International Conference on 2010, page(s): 330 - 333.

[9] B.Ramakrishnan, Dr.R.S.Rajesh, R.S.Shaji, “An Intelligent Routing Protocol for Vehicle safety communication in Highway Environments", accepted for publication, journal of computing-Volume 2, Issue 11, November 2010dec 2010.

[10] Qi Chen ,Felix Schmidt Eisenlohr, DanielJiang, Marc Tollent-Moreno-Luca Delgrossi "Overhaul of ieee 802.11 modeling and simulation in ns-2" ACM New York, NY, USA -2007, Pages: 159 - 168.

[11] Nakjung Choi Sungjoon Choi Yongho Seok Taekyoung Kwon Yanghee "A Solicitation-based IEEE 802.11p MAC Protocol for Roadside to Vehicular Networks", 2007 Mobile Networking for Vehicular Environments, May 2007, Pages 91-96

[12] B.Ramakrishnan,R.S.Rajesh,R.S.Shaji,” Performance Analysis of 802.11 and $802.11 \mathrm{p}$ in Cluster Based Simple Highway Model", IJCSIT, volume 1 issue 5 Nov 2010, page:420-426.

[13] Berijesh Kadri Mohandas, Amiya Nayak, Kshirasagar Naik, Nishith Goel "ABSRP: A Service Discovery Approach for Vehicular Ad-Hoc Networks" page 1590-1594 IEEE Asia Pacific Service Computing Conference 2008. 
International Journal of Next-Generation Networks (IJNGN) Vol.2, No.4, December 2010

[14] Zuo, Jing Wang, Yuhao Liu, Yuan Zhang, Yan," Performance Evaluation of Routing Protocol in VANET with Vehicle-Node Density", (WiCOM), 2010 ,onOn page(s): 1 - 4.

[15] Wisitpongphan, N. Tonguz, O.K. Parikh, J.S. Mudalige, P. Bai, F. Sadekar, V. Carnegie Mellon Univ., Pittsburgh "Broadcast storm mitigation techniques in vehicular ad hoc networks" Wireless Communications, IEEE-December2007,Volume:14,Issue:6, pages : 84-94.

[16] Boban, M. Tonguz, O.K. Barros, J. "Unicast communication in vehicular ad hoc networks: a reality check" IEEE Issue Date : December 2009 Volume : 13, Issue:12 On page(s): 995-997.

[17] Shi, W. Liu Y "Real-Time urban traffic monitoring with global positioning System equipped vehicles" Intelligent Transport System, IET, Issue Date: June 2010, Volume 4 Issue 2 Page :113-120.

[18] Denko, M.K. Jun Tian Nkwe, T. Obaidat, M.S. "Cluster-Based Cross-Layer Design for Cooperative Caching in Mobile Ad Hoc Networks", Systems Journal, IEEE, Issue Date : Dec. 2009 ,Volume : 3 , Issue:4 ,On page(s): 499 - 508.

[19] L.Bononi M. Di Felice, " A Cross Layered MAC and Clustering Scheme for efficient Broadcast in VANETs” IEEE MASS'07 page 1-8,October 2007,Pisa, Italy.

[20] Ivan, I. Besnier, P. Crussiere, M. Drissi, M. Le Danvic, L. Huard, M. Lardjane, E. Technocentre Renault, Guyancourt, France "Physical layer performance analysis of V2V communications in high velocity context" Intelligent Transport Systems Telecommunications,(ITST),2009 9th International Conference, Oct 2009,Pages 409 .

[21] B.Ramakrishnan,R.S.Rajesh,R.S.Shaji," CBVANET: A Cluster Based Vehicular Adhoc Network Model for Simple Highway Communication", Int.J.Advanced Networking and Application Dec-2010 ,accepted for publication.

[22] Schoch, E. Kargl, F. Weber, M. Leinmuller, T. "Communication patterns in VANETs" Communications Magazine, IEEE Issue Date : November 2008 Volume : 46, Issue:11 On page(s): 119 - 125 Canada

\section{Authors}

B. Ramakrishnan is currently working as Associate Professor in the Department of Computer Science in S.T. Hindu College, Nagercoil. He received his MSc degree from Madurai Kamaraj university, Madurai and received Mphil (comp Sc) from Alagappa University Karikudi. He has a teaching experience of 23 years. His research interests lie in the field of Vehicular networks, mobile networks and Ad-hoc networks.

Dr. R. S Rajesh received his B.E and M.E degrees in Electronics and Communication Engineering from Madurai Kamaraj University, Madurai, India in the year 1988 and 1989 respectively, In September 1992 he joined as a faculty member in the Department of Computer Science and Engineering, Manonmaniam Sundaranar University where he earned his Doctorate degree in the field of Computer Science and Engineering. He is currently working as Associate Professor in the Department of Computer Science and Engineering. He got more than 20 years of PG teaching and Research experience. His current research areas include Vehicular Networks, Wireless networks, Digital image processing, Pervasive computing.

Mr. R S Shaji received his MCA degree from Manonmaniam Sundaranar University, Tirunelveli and M.Tech. in Computer Science and Engineering from Pondicherry University. $\mathrm{He}$ is currently working as Assistant Professor and Head of Department of Computer Applications in St.Xavier's catholic college of Engineering, Kanyakumari district. He is doing his research in Manonmaniam Sundaranar University, Tirunelveli, India. His research interests are Service Discovery and routing in Mobile Adhoc networks, Pervasive Applications and High Performance Computing
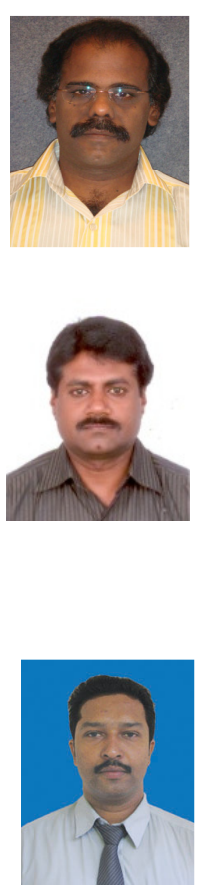